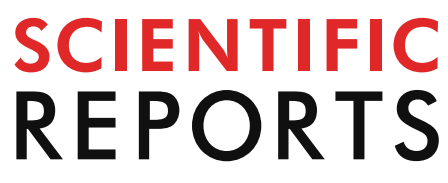

natureresearch

Check for updates

\title{
Development of fluorescent Escherichia coli for a whole-cell sensor of 2'-fucosyllactose
}

\author{
Jonghyeok Shin ${ }^{1,2}$, Myungseo Park ${ }^{1}$, Chakhee Kim ${ }^{1}$, Hooyeon Kim ${ }^{1}$, Yunjeong Park ${ }^{1}$, \\ Choongjin Ban ${ }^{1,3}$, Jong-Won Yoon ${ }^{4}$, Chul-Soo Shin ${ }^{4}$, Jae Won Lee ${ }^{2,5}$, Yong-Su Jin ${ }^{2,5}$, \\ Yong-Cheol Park ${ }^{6}$, Won-Ki Min ${ }^{7 凶}$ \& Dae-Hyuk Kweon ${ }^{1,3,8 \boxplus ~}$
}

2'-Fucosyllactose (2'-FL), a major component of fucosylated human milk oligosaccharides, is beneficial to human health in various ways like prebiotic effect, protection from pathogens, anti-inflammatory activity and reduction of the risk of neurodegeneration. Here, a whole-cell fluorescence biosensor for 2'-FL was developed. Escherichia coli (E. coli) was engineered to catalyse the cleavage of 2'-FL into L-fucose and lactose by constitutively expressing $\alpha$-L-fucosidase. Escherichia coli $\Delta$ L YA, in which lacZ is deleted and lacY is retained, was employed to disable lactose consumption. $E$. coli $\Delta L$ YA constitutively co-expressing $\alpha$-L-fucosidase and a red fluorescence protein (RFP) exhibited increased fluorescence intensity in media containing 2'-FL. However, the presence of $50 \mathrm{~g} / \mathrm{L}$ lactose reduced the RFP intensity due to lactose-induced cytotoxicity. Preadaptation of bacterial strains to fucose alleviated growth hindrance by lactose and partially recovered the fluorescence intensity. The fluorescence intensity of the cell was linearly proportional to $1-5 \mathrm{~g} / \mathrm{L} \mathrm{2'-FL.} \mathrm{The} \mathrm{whole-cell} \mathrm{sensor} \mathrm{will} \mathrm{be} \mathrm{versatile} \mathrm{in} \mathrm{developing}$ a $2^{\prime}-$ FL detection system.

Human milk oligosaccharides (HMOs) are present in human breast milk and are closely associated with health benefits. HMOs act as decoys for pathogens (e.g., virus, bacteria, and protozoa) by inhibiting their ability to bind to the surface of epithelial cells ${ }^{1-4}$. Fucosylated oligosaccharides which are not found in bovine milk account for $50 \%$ of total HMOs. The HMOs 2'-fucosyllactose (2'-FL; Fuc- $\alpha 1,2-G a l-\beta 1,4-G l c)$ and 3-fucosyllactose (3-FL; Gal- $\beta 1,4-F u c-\alpha 1,3-G l c)$, with a concentration range of $0.5-2 \mathrm{~g} / \mathrm{L}$, account for the largest portion of fucosylated $\mathrm{HMOs}^{5,6}$. Fucosylated HMOs circulate systemically, affecting the host immune response, the regulation of tumour metastasis, and resistance to bacteria, fungi, and other pathogens ${ }^{2,7,8}$. The concentration of $2^{\prime}$-FL in breast milk affects the ability to protect against vital systemic infections in nursing infant ${ }^{8}$. While low content of $2^{\prime}$-FL in breast milk has been associated with a higher rate of diarrhoea during lactation ${ }^{9,10}$, about $20 \%$ of human milk do not contain 2'-FL ${ }^{11}$. For these reasons, 2'-FL and 3-FL are spotlighted as nutraceutical and pharmaceutical ingredients; thus, a simple and visually measurable method is indispensable in evaluating their level in breast milk.

Various methods have been developed for the production of $2^{\prime}$-FL, including whole-cell biocatalysis ${ }^{12-20}$, enzymatic synthesis ${ }^{21,22}$, and chemical synthesis ${ }^{23,24}$. Regardless of the method used, a simple detection and quantification method is indispensable for the development of $2^{\prime}$-FL production. Quantification of $2^{\prime}$-FL has been mostly done using high-performance liquid chromatography (HPLC), high-pH anion exchange chromatography, and liquid chromatography-mass spectrometry (LC-MS) ${ }^{15,25-28}$. However, those types of equipment are time-consuming, labour-intensive, and expensive. Therefore, those methods are unlikely to be used in a high-throughput manner or for a brief test.

Recently, we developed 2 '-FL quantification assays through two-step enzymatic reaction ${ }^{19}$ or one-pot reaction ${ }^{20}$ of fucosidase and fucose dehydrogenase, where $2^{\prime}$-FL concentration is spectroscopically readout by

\footnotetext{
${ }^{1}$ Department of Integrative Biotechnology, College of Biotechnology and Bioengineering, Sungkyunkwan University, Suwon 16419, Republic of Korea. ${ }^{2}$ Carl R. Woese Institute for Genomic Biology, University of Illinois at Urbana-Champaign, Urbana, IL 61801, USA. ${ }^{3}$ Biomedical Institute for Convergence, Sungkyunkwan University, Suwon 16419, Republic of Korea. ${ }^{4}$ AP Technology, Suwon 16229, Republic of Korea. ${ }^{5}$ Department of Food Science and Human Nutrition, University of Illinois At Urbana-Champaign, Urbana, IL 61801, USA. ${ }^{6}$ Department of Bio and Fermentation Convergence Technology, Kookmin University, Seoul 02707, Republic of Korea. ${ }^{7}$ Department of Food Science and Industry, Kyungil University, Gyeongsan 38428, Republic of Korea. ${ }^{8}$ Center for Biologics, Sungkyunkwan University, Suwon 16419, Republic of Korea. ${ }^{\varpi}$ email: elvptl76@gmail.com; dhkweon@skku.edu
} 
A
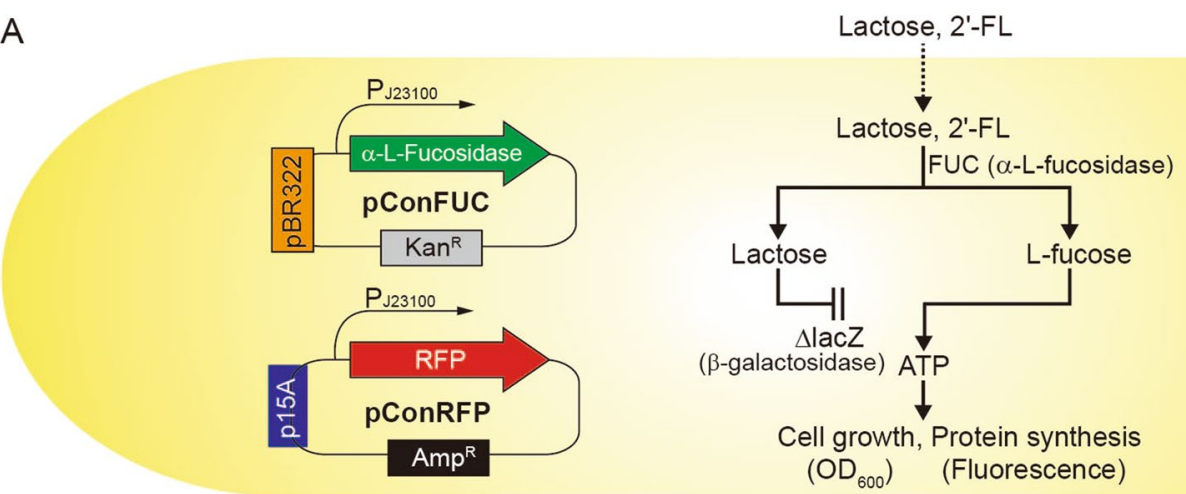

B

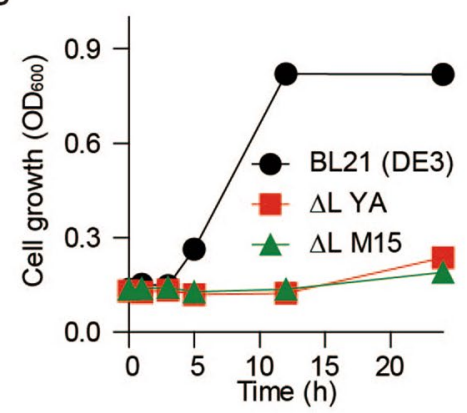

C

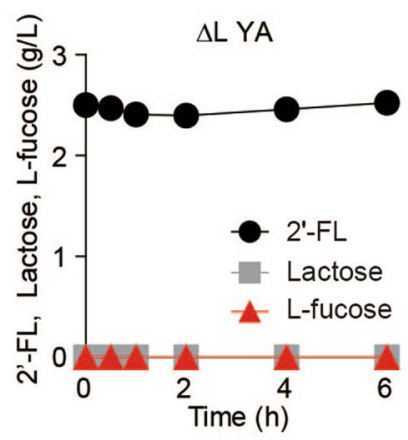

D

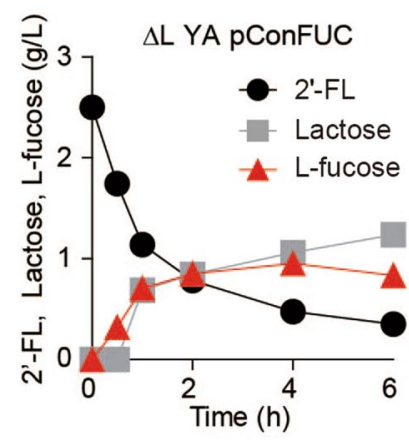

Figure 1. Schematic of a fluorescent E. coli 2'-FL biosensor. (A) Digestion of 2'-FL by a-L-fucosidase in $E$. coli. $2^{\prime}-\mathrm{FL}$ is cleaved to $\mathrm{L}$-fucose and lactose by $\alpha$-L-fucosidase. When $l a c Z$ is deleted, L-fucose released from $2^{\prime}$-FL is the sole carbon source for cell growth and fluorescence protein expression. Schematic was designed using Adobe Illustrator CS6 (v16.0.0, Adobe Systems Inc., USA). (B) Growth of E. coli strains using lactose as a carbon source. E. coli strains were cultured in R medium, which included $2 \mathrm{~g} / \mathrm{L}$ lactose. Symbols denote the cell densities of E. coli BL21 (DE3) (filled circle), $\triangle \mathrm{L}$ YA (filled square), and $\triangle \mathrm{L}$ M15 (filled upward traingle). Cleavage of 2'-FL by soluble lysates of (C) E. coli $\triangle \mathrm{L} Y A$ and (D) E. coli $\triangle \mathrm{L}$ YA pConFUC. Symbols denote the concentrations of $2^{\prime}$-FL (filled circle), Lactose (filled square), and L-fucose (filled upward traingle).

reduction of NADPH. In this study, we developed a visual detection method for 2'-FL using an E. coli strain expressing an $\alpha$-L-fucosidase (FUC, E.C. 3.2.1.63) and a red fluorescence protein (RFP), which can be used in high-throughput applications. L-fucose was released from 2'-FL inside the bacterial cell by $\alpha$-L-fucosidase, which specifically catalyses the hydrolysis of a1-2-linked L-fucopyranosyl residues from various oligosaccharides ${ }^{29}$. L-fucose was then used for RFP synthesis (Fig. 1A). The lacZ-deficient E. coli strain was used to disable lactose consumption by cells. This engineered strain semi-quantitatively reported the 2'-FL content in milk in highthroughput by emitting fluorescence in a 2 '-FL concentration-dependent manner.

\section{Results}

Evaluating 2'-FL-dependent cell growth and fluorescence using a whole-cell biosensor. A whole-cell biosensor, which grows and emits fluorescence only in the presence of 2'-FL, was designed (Fig. 1A). The plasmid pConFUC was constructed to constitutively express $\alpha$-L-fucosidase under the control of the J23100 promoter (Fig. 1A). The 2'-FL can be hydrolysed by a-L-fucosidase in E. coli so that the amount of released L-fucose and lactose are proportional to $2^{\prime}$-FL concentration. The cells were engineered not to metabolize lactose by deleting their endogenous $\beta$-galactosidase gene (lacZ) while retaining lac $Y$ to import 2'-FL. Two E. coli BL21 (DE3) variants, $\triangle \mathrm{L} Y \mathrm{YA}$ and $\Delta \mathrm{L}$ M15, with different levels of residual $\beta$-galactosidase activity, were cultured in $\mathrm{R}$ medium containing $2 \mathrm{~g} / \mathrm{L}$ lactose ${ }^{13,16}$. Both variants did not grow with lactose as a sole carbon source in a minimal medium for $24 \mathrm{~h}$, whereas wild-type E. coli BL21 (DE3) grew well using lactose (Fig. 1B). The difference of growth rate of $\Delta \mathrm{L}$ M15 and $\Delta \mathrm{L}$ YA in lactose was marginal. For the strict restriction of lactose consumption, the E. coli $\Delta \mathrm{L}$ YA strain which has much lower $\beta$-galactosidase activity was used in the following studies ${ }^{13,16}$.

The active expression of $\alpha$-L-fucosidase in E. coli $\triangle \mathrm{L}$ YA was examined by analysing the 2'-FL cleavage in the soluble fraction of E. coli $\Delta$ L YA cell extracts. The cell extracts of wild type E. coli BL21 (DE3) and E. coli $\Delta \mathrm{L}$ YA (Fig. S1A and Fig. 1C) did not digest 2'-FL. In contrast, 2'-FL was rapidly digested to release lactose and L-fucose by the cell extracts of E. coli BL21 (DE3) pConFUC (Fig. S1B) and E. coli $\triangle$ L YA pConFUC (Fig. 1D). These results suggest that $\alpha$-L-fucosidase was actively expressed in $E$. coli regardless of lac $Z$ deletion.

$2^{\prime}$-FL detection by the increase in fluorescence intensity. E. coli $\triangle \mathrm{L}$ YA or $\triangle \mathrm{L}$ YA pConFUC were inoculated with $\mathrm{R}$ medium containing no carbon source, $2 \mathrm{~g} / \mathrm{L} 2^{\prime}$-FL, $2 \mathrm{~g} / \mathrm{L}$ lactose, or a mixture of $2 \mathrm{~g} / \mathrm{L} 2^{\prime}$-FL 
A

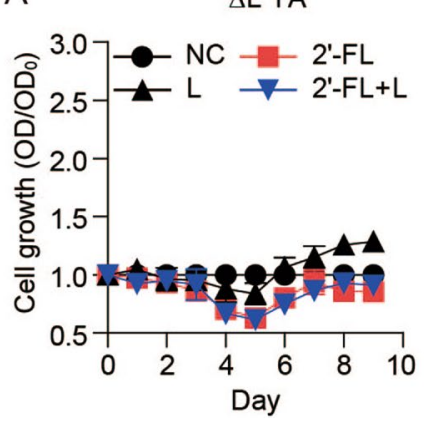

B

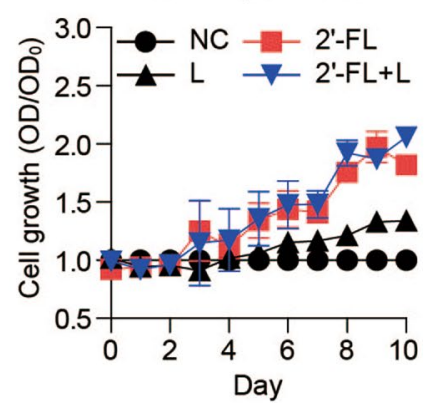

C

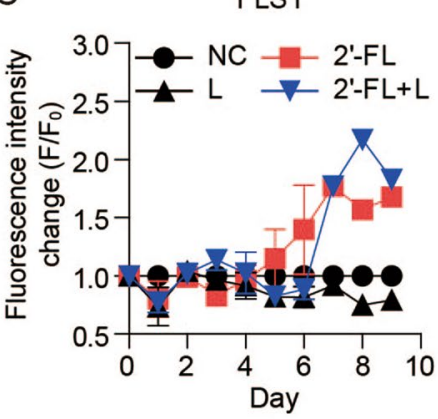

Figure 2. Detection of $2^{\prime}$-FL through RFP fluorescence of $E$. coli strains. E. coli $\Delta \mathrm{L}$ YA, $\triangle \mathrm{L}$ YA pConFUC, and FLS1 strains were cultured in R medium, which contained lactose, 2'-FL or mixture of lactose and 2'-FL. Relative optical density (ROD) of (A) E. coli $\Delta$ L YA, (B) E. coli $\Delta$ L YA pConFUC, (C) Fluorescence intensity change $\left(\mathrm{F} / \mathrm{F}_{0}\right)$ of RFP in FLS1 (E. coli $\triangle \mathrm{L}$ YA pConFUC/pConRFP). Symbols denote the negative control (filled circle), $2 \mathrm{~g} / \mathrm{L} 2^{\prime}$-FL (filled square), $2 \mathrm{~g} / \mathrm{L}$ lactose (filled upward traingle), and $2 \mathrm{~g} / \mathrm{L}$ lactose $+2 \mathrm{~g} / \mathrm{L} 2^{\prime}$-FL (filled downward triangle). Results are the average of biological replicates $(n=2)$. Error bars represent standard deviations and are not displayed when smaller than symbol size.

and $2 \mathrm{~g} / \mathrm{L}$ lactose. E. coli $\Delta \mathrm{L}$ YA did not grow with any carbon source while E. coli $\Delta \mathrm{L}$ YA pConFUC grew slowly in the presence of $2^{\prime}$-FL (Fig. 2A,B, Table S2) and did not grow using lactose as a sole carbon source. However, lactose did not affect the cell growth of $E$. coli $\triangle \mathrm{L}$ YA pConFUC using 2 '-FL as the carbon source (Fig. 2B).

E. coli $\triangle \mathrm{L}$ YA pConFUC was co-transformed with pConRFP expressing a red fluorescence protein (RFP) under the control of the $\mathrm{J} 23100$ promoter. E. coli $\triangle \mathrm{L}$ YA pConFUC/pConRFP (FLS1) cells had a long lag period of 4 days after their inoculation with R medium containing $2 \mathrm{~g} / \mathrm{L} 2^{\prime}$-FL or a mixture of 2'-FL and lactose. Cells did not grow in R medium containing only lactose. RFP signal began to increase on the fifth day of bacterial inoculation in R medium containing 2'-FL (Fig. 2C). Although FLS1 showed a 2'-FL-dependent cell growth and a fluorescence increase, there was a few-days of long lag period. We thought that the cell was not ready to metabolize fucose even after 2 '-FL cleavage to fucose and lactose.

Adaptation of the whole-cell biosensor to L-fucose for faster response toward 2'-FL. As the L-fucose metabolic pathway should be activated to use $\mathrm{L}$-fucose as a carbon source ${ }^{30}$, we tested whether the delayed response can be resolved by pre-adapting cells to L-fucose to shorten the lag period. Cells precultured in LB broth showed a long lag period lasting a day in R medium containing L-fucose (Fig. $3 \mathrm{~A}$ ). When E. coli $\Delta \mathrm{L}$ YA was preadapted in R medium containing $10 \mathrm{~g} / \mathrm{L}$ of L-fucose before inoculation for several rounds, the lag period was dramatically shortened, and the cells immediately entered the exponential growth phase upon inoculation (Fig. 3B). When adapted cells were precultured in the LB broth a second time, cell growth on the L-fucose was retarded similarly to the non-adapted cells (Fig. 3C). These results suggest that this fast-growing phenotype arose because of simple adaptation to the substrate.

E. coli $\triangle \mathrm{L}$ YA pConFUC/pConRFP preadapted to L-fucose (denoted as FLS2) was cultured in R medium containing $2 \mathrm{~g} / \mathrm{L} \mathrm{L}$-fucose. While $E$. coli $\Delta \mathrm{L}$ YA lacking the plasmid for fucosidase did not grow in any media for 4 days, FLS2 showed exponential growth after a short lag period ( $<8 \mathrm{~h}$; Fig. 3D,E). Notably, the RFP fluorescence reached its maximal fluorescence within 10-20 h when FLS2 was grown in R medium containing 2'-FL (Fig. 3F). The fluorescence emission lasted for 4 days at a stable emission. Cells did not emit fluorescence using lactose as a sole carbon source and $2 \mathrm{~g} / \mathrm{L}$ lactose present together with $2^{\prime}$-FL did not alter fluorescence intensity either. Although RFP intensity increased by only $~ 50 \%$, these results suggest that RFP fluorescence was a relatively fast and reliable reporter of $2^{\prime}$-FL.

Enhanced fluorescence intensity. To quicken the $2^{\prime}$-FL detection and increase fluorescence intensity, the expression cassettes of RFP and fucosidase were recombined using different vectors. The small copy number (10-12) replicon p15A was replaced by the high copy number (20-40) replicon ColA for RFP expression (Fig. 4a) ${ }^{31}$. The plasmid pET-ConFUC was also constructed by replacing the kanamycin resistance cassette of pConFUC with the ampicillin resistance cassette of pET (Fig. $4 \mathrm{~B}$ and Table 1). The E. coli $\Delta \mathrm{L}$ YA strain was then transformed with the resulting plasmids pET-ConFUC and pColA-ConRFP. When E. coli $\triangle \mathrm{L}$ YA pETConFUC/pColA-ConRFP (FLS3) was inoculated with R medium containing 2'-FL, fluorescence increased after a short lag phase $(<2$ h; Fig. $4 \mathrm{C})$. Furthermore, the maximum fluorescence intensity change was $\sim 7$ times higher than previous transformants containing pConRFP. The new whole-cell sensor not only exhibited much stronger fluorescence than the previous one the fluorescence intensity change was proportional to a 2 '-FL concentration of 1-5 g/L (Fig. 4D and Fig. S2). The signal was strong enough to enable the visualization of the fluorescence (Fig. 4E).

Reduced lactose toxicity by fucose adaptation. We observed that the enhancement of fluorescence intensity by the new combination of expression cassettes was hindered by the presence of only $2 \mathrm{~g} / \mathrm{L}$ lactose 
A

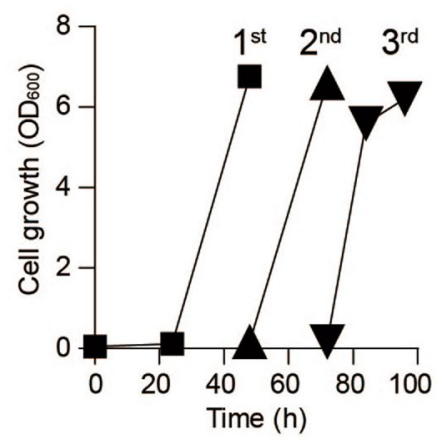

D

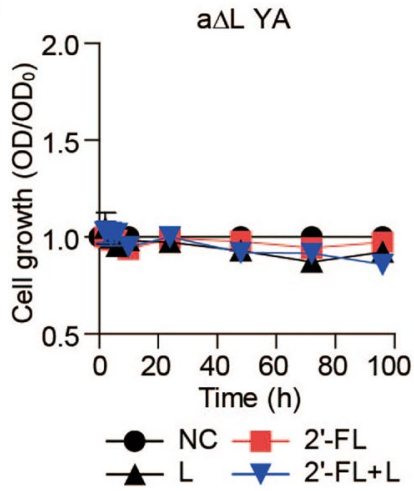

B

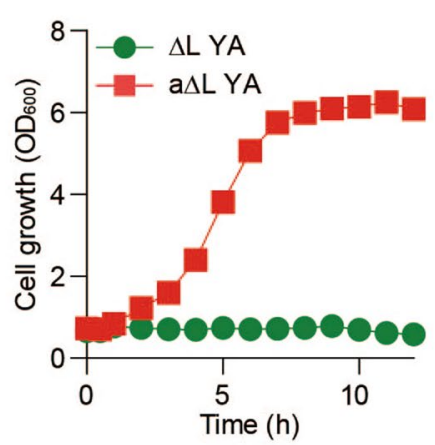

E

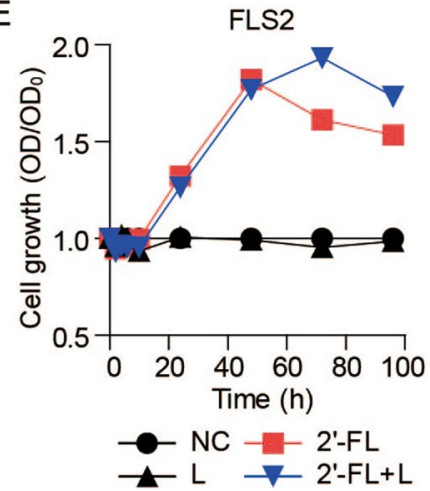

C

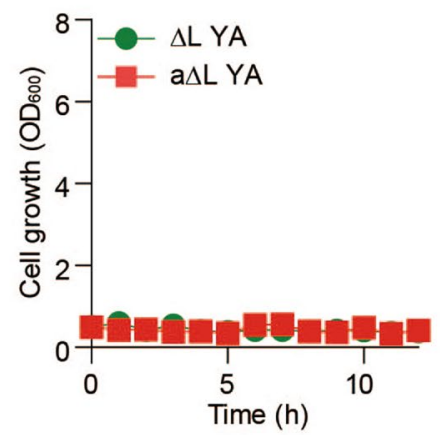

$\mathrm{F}$

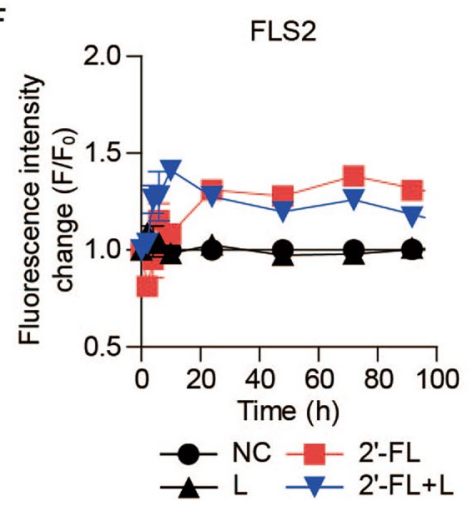

Figure 3. Adaptation of E. coli $\triangle \mathrm{L} Y A$ to the L-fucose. (A) Repeated culture of E. coli $\triangle \mathrm{L}$ YA for adaptation to L-fucose. (B) Growth curves of $\triangle \mathrm{L}$ YA and adapted $\triangle \mathrm{L}$ YA (a $\triangle \mathrm{L}$ YA) in R medium containing $10 \mathrm{~g} / \mathrm{L} \mathrm{L}$-fucose after preculture in L-fucose-containing R medium. $\triangle \mathrm{L}$ YA (filled circle); $\triangle \Delta \mathrm{L}$ YA (filled square). (C) Growth curves of $\triangle \mathrm{L} Y A$ and a $\triangle \mathrm{L}$ YA in $\mathrm{R}$ medium containing $10 \mathrm{~g} / \mathrm{L} \mathrm{L}$-fucose after preculture in $\mathrm{LB} . \Delta \mathrm{L}$ YA (filled circle); a $\triangle \mathrm{L}$ YA (filled square). (D) Cell growth of a $\triangle \mathrm{L}$ YA in R medium containing 2'-FL and/or lactose. (E) Cell growth of FLS2 in R medium containing 2'-FL and/or lactose. (F) Fluorescence intensity change (F/F $\mathrm{F}_{0}$ ) of RFP in FLS2 (E. coli $\triangle \mathrm{L}$ YA pConFUC/pConRFP preadapted to L-fucose). Negative control (filled circle), $2 \mathrm{~g} / \mathrm{L} 2$ '-FL (filled square), $2 \mathrm{~g} / \mathrm{L}$ lactose (filled upward traingle), and $2 \mathrm{~g} / \mathrm{L}$ lactose $+2 \mathrm{~g} / \mathrm{L} 2^{\prime}$-FL (filled downward triangle). Results are the average of biological replicates $(n=2)$. Error bars represent standard deviations and are not displayed when smaller than symbol size $(\mathbf{d}-\mathbf{f})$.

(Fig. 4C). Indeed, media containing $50 \mathrm{~g} / \mathrm{L}$ lactose or bovine milk supplemented with 2'-FL showed weak fluorescence intensity (Fig. 5B), indicating the hurdles imposed by lactose-induced cytotoxicity.

E. coli symports lactose and protons through $\operatorname{lac} Y$, the lactose permease structural gene, and acidifies the cytoplasm via lactose transport. This acidification causes cytotoxicity followed by the induction of cellular acid shock, resulting in the reduction of proton motive force, intracellular ATP levels, and cell viability ${ }^{32}$. As mentioned above, to accelerate fucose use, preadaptation to fucose was applied a second time to relieve the negative effects of cytoplasmic acidification by lactose. E. coli symports $\mathrm{L}$-fucose and $\mathrm{H}^{+}$by a $\mathrm{L}$-fucose $/ \mathrm{H}^{+}$symporter, and the internalized cytoplasmic proton would induce $E$. coli to activate its acid resistance system and relieve cytoplasmic acidification ${ }^{33}$. Therefore, preadaptation to fucose can be used not only to activate L-fucose metabolic pathway but also to enhance acid resistance by bacterial cells (Fig. 5A). Indeed, when $\triangle \mathrm{L}$ YA pET-ConFUC/ pColA-ConRFP was preadapted to fucose (denoted as FLS4), $2 \mathrm{~g} / \mathrm{L}$ lactose did not hinder the 2'-FL detection (Fig. 5C). Furthermore, pre-adaption to fucose enabled much larger fluorescence intensity change than the nonadapted cells, even in the presence of $50 \mathrm{~g} / \mathrm{L}$ lactose (Fig. 5D,E). Pre-adaption no longer improved the fluorescence intensity when $2^{\prime}$-FL was added to bovine milk. However, the fluorescence intensity was still proportional to 2'-FL concentration (Fig. 5F). Both biosensors were still able to detect the biologically relevant concentration of $2^{\prime}$-FL mixed in bovine milk (Fig. S3).

\section{Discussion}

We designed and developed a functional E. coli whole-cell 2'-FL biosensor based on the growth-coupled red fluorescence emission produced after the cleavage of $2^{\prime}$-FL by recombinant $\alpha$-L-fucosidase, and we demonstrated that the biosensor could quantify $2^{\prime}$-FL. As the working principle of the biosensor, $2^{\prime}$-FL enters E. coli $\Delta \mathrm{L}$ YA perhaps via $l a c Y$ and then is hydrolysed into $\mathrm{L}$-fucose and lactose by the recombinant $\alpha$-L-fucosidase in the cytosolic space. Because lactose is an abundant disaccharide in milk, it can be used after hydrolysing into glucose and galactose by $\beta$-galactosidase in the wild-type E. coli. Therefore, endogenous lac $Z$ was deleted completely $(\Delta \mathrm{L}$ YA) or partially disrupted ( $\triangle \mathrm{L} \mathrm{M} 15)$. We confirmed that both mutants did not grow in lactose medium at all, and there was no red fluorescence emitted in $\triangle \mathrm{L}$ YA in the absence of a carbon source (Fig. 1B). Among the E. coli mutants, 
A

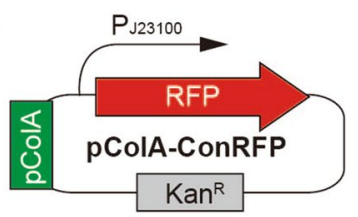

B

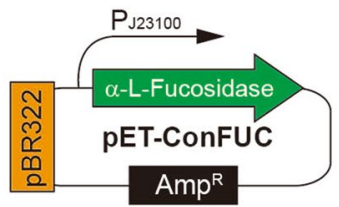

C

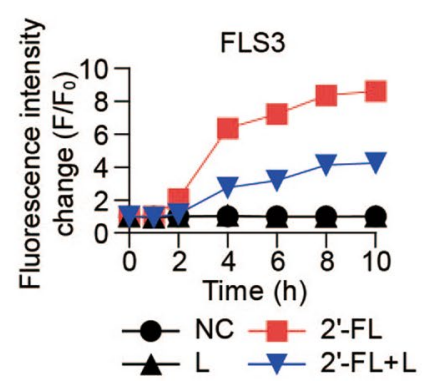

D

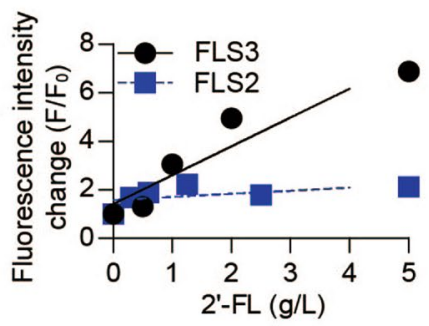

$\mathrm{E}$

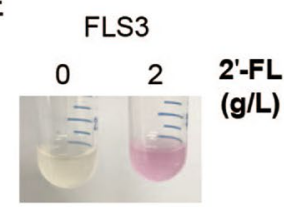

Figure 4. Improved 2 '-FL detection. (A) Plasmid pColA-ConRFP for enhancing RFP expression. (B) Plasmid pET-ConFUC for $\alpha$-L-fucosidase expression. (C) Fluorescence intensity change (F/F $\mathrm{F}_{0}$ ) of RFP in FLS3 (E. coli $\Delta \mathrm{L}$ YA pET-ConFUC/pColA-ConRFP). Symbols denote negative control (filled circle), 2 g/L 2'-FL (filled square), $2 \mathrm{~g} / \mathrm{L}$ lactose (filled upward traingle), and $2 \mathrm{~g} / \mathrm{L}$ lactose $+2 \mathrm{~g} / \mathrm{L} 2^{\prime}$-FL (filled downward triangle). (D) Comparison of FLS2 and FLS3 for 2'-FL-dependent fluorescence response. FLS3, $\triangle \mathrm{L}$ YA pET-ConFUC/pColA-ConRFP (filled circle); FLS2, a $\triangle$ L YA pConFUC/pConRFP (filled square). (C, D) Results are the average of biological replicates $(n=3)$. Error bars represent standard deviations and are not displayed when smaller than symbol size. (E) A picture of FLS3 cultured in the presence or absence of 2'-FL for $10 \mathrm{~h}$ (Left, R media only; Right, R media containing $2 \mathrm{~g} / \mathrm{L} 2^{\prime}$-FL). The representative image from biological replicates $(n=3)$ was processed using Adobe Illustrator CS6 (v16.0.0, Adobe Systems Inc., USA).

\begin{tabular}{|l|l|l|}
\hline Strain/plasmid & Relevant description & References \\
\hline E. coli Top10 & $\begin{array}{l}\text { F-mcrA } \Delta \text { (mrr-hsdRMS-mcrBC) } \Phi 80 \text { lacZ } \Delta \text { M15 } \Delta \text { lacX74 recA1 araD139 } \Delta \text { (ara leu) } 7,697 \text { galU galK } \\
\text { rpsL (StrR) endA1 nupG }\end{array}$ & Invitrogen \\
\hline$\Delta$ L YA & BL21star (DE3) $\Delta$ L Tn7::lacYA (lacZ deleted) & 13 \\
\hline$\Delta$ L M15 & BL21star (DE3) $\Delta$ lacZYA Tn7::lacZ $\Delta$ M15 (lacZ disrupted) & 16 \\
\hline FLS1 & DL YA harbouring pConFUC, pConRFP & This study \\
\hline FLS2 & Fucose-adapted FLS1 & This study \\
\hline FLS3 & $\Delta$ L YA harbouring pET-ConFUC, pColA-ConRFP & This study \\
\hline FLS4 & Fucose-adapted FLS3 & This study \\
\hline pConFUC & J23100 constitutive promoter/express $\alpha$-L-fucosidase/kanamycin resistance/pBR322 replication origin & This study \\
\hline pET-ConFUC & J23100 constitutive promoter/express $\alpha$-L-fucosidase/ampicillin resistance/pBR322 replication origin & This study \\
\hline pConRFP & J23100 constitutive promoter/express RFP/ampicillin resistance/p15A replication origin & This study \\
\hline pColA-ConRFP & J23100 constitutive promoter/express RFP/kanamycin resistance/pColA replication origin & This study \\
\hline
\end{tabular}

Table 1. List of strains and plasmids used in this study.

the $\triangle \mathrm{L}$ YA strain was chosen as the backbone strain for the transformation with the $\alpha$-L-fucosidase originating from Xantomonas manhihotis. The a-L-fucosidase was active in E. coli cytoplasm and efficiently cleaved the a $(1 \rightarrow 2)$ L-fucose branched site in the trisaccharide 2'-FL and hydrolysed it into lactose and L-fucose (Fig. 1D). L-fucose could be consumed, even though small amount, by E. coli cells extracts suggesting that L-fucose can be used as a sole carbon source. The expression of recombinant $\alpha$-L-fucosidase and complete deletion of lac $Z$ allowed cell growth in 2'-FL-containing media (Fig. 2B).

After 2'-FL hydrolysis inside the E. coli cell, L-fucose, a hexose, is metabolized into dihydroxyacetone phosphate and L-lactaldehyde by the sequential actions of a permease, an isomerase, a kinase, and an aldolase $\mathrm{e}^{30}$. Aerobically, L-lactaldehyde is oxidized in two steps to pyruvate using NAD-dependent lactaldehyde dehydrogenase and flavin-linked lactate dehydrogenase, and thus channelling all carbons from L-fucose into the central metabolic pathways involved in ATP and amino acid synthesis ${ }^{30}$. Preadaptation to fucose is likely to accelerate cell growth and RFP production because this pathway is activated.

Lactose causes cytotoxicity in several ways. When lactose is transported into a cell that cannot metabolize lactose, the cellular membrane is damaged, and the membrane potential is disrupted by the so-called lactosekilling effect ${ }^{34}$. Lactose can induce cytoplasmic acidification as it is transported into the cytoplasm with a proton through the lactose permease. The resulting acidification of the cytoplasm induces cellular acid shock and reduces 
A

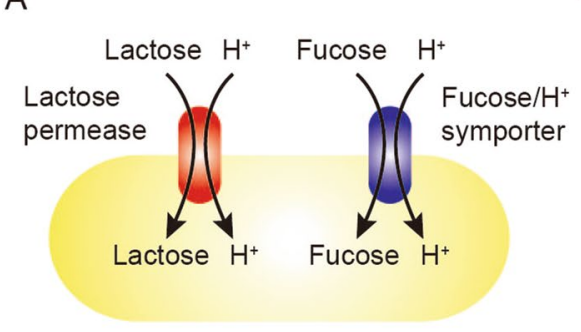

B

E

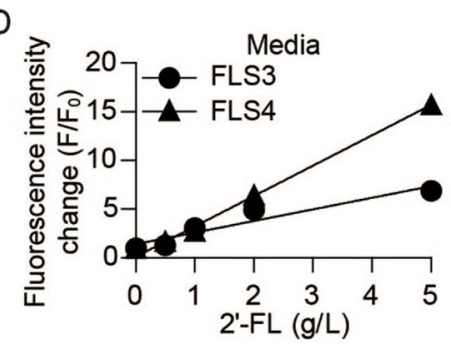

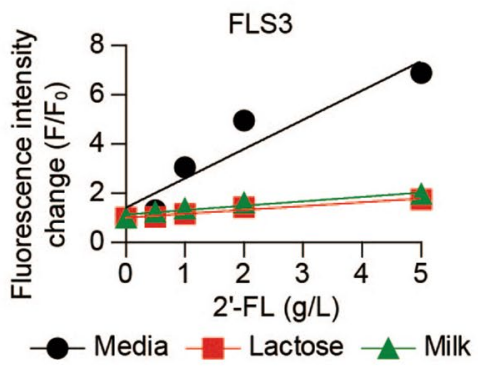

Media - Lactose $\rightarrow$ Milk
C

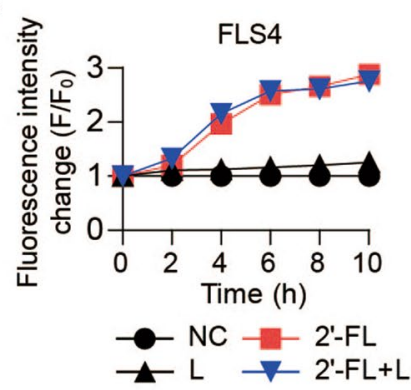

$\mathrm{F}$

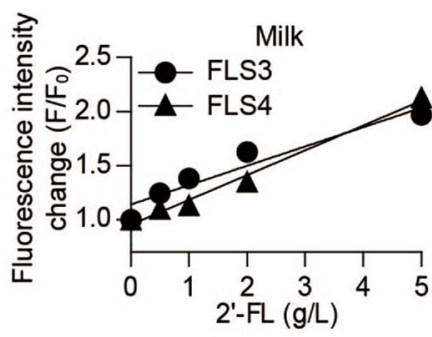

Figure 5. Pre-adaption of the biosensor to fucose to overcome lactose-induced cytotoxicity. (A) Acid adaptation with fucose. Lactose permease symports protons as well as lactose lowering intracellular $\mathrm{pH}$. The fucose $/ \mathrm{H}^{+}$symporter also symports fucose and proton ${ }^{33}$. Fucose adaptation induces not only a fucose assimilation pathway but also the acid tolerance mechanism. Schematic was designed using Adobe Illustrator CS6 (v16.0.0, Adobe Systems Inc., USA). (B) Fluorescence intensity of FLS3 decreased in the presence of lactose. FLS3 ( $\triangle \mathrm{L}$ YA pET-ConFUC/pColA-ConRFP) was supplemented with $2 \mathrm{~g} / \mathrm{L} 2^{\prime}$-FL (filled circle), $2 \mathrm{~g} / \mathrm{L}$ $2^{\prime}-\mathrm{FL}+50 \mathrm{~g} / \mathrm{L}$ Lactose (filled square), and $2 \mathrm{~g} / \mathrm{L} 2^{\prime}-\mathrm{FL}+$ bovine milk (filled upward traingle). Fluorescence intensity was measured after $10 \mathrm{~h}$ incubation. (C) FLS4 (a $\triangle \mathrm{L}$ YA pET-ConFUC/pColA-ConRFP), which was preadapted to fucose, was cultured in the presence or absence of lactose and 2'-FL. Symbols denote negative controls (filled circle), $2 \mathrm{~g} / \mathrm{L} 2^{\prime}$-FL (filled square), $2 \mathrm{~g} / \mathrm{L}$ lactose (filled upward traingle), and $2 \mathrm{~g} / \mathrm{L}$ lactose $+2 \mathrm{~g} / \mathrm{L}$ 2'-FL (filled downward triangle). (D-F) Comparison of FLS3 and FLS4 for 2'-FL-dependent fluorescence response. (D) Fluorescence intensity became stronger when the cells were preadapted to fucose. (E) FLS4 exhibiting higher fluorescence intensity than the non-adapted cell in the presence of $50 \mathrm{~g} / \mathrm{L}$ lactose. (F) The increase in the fluorescence intensity proportional to 2'-FL when supplemented in bovine milk. Average from biological replicates is shown $(n=3)$. Error bars represent standard deviations and are not displayed when smaller than symbol size (B-F).

proton motive force, intracellular ATP levels, and cell viability ${ }^{32}$. Our results show that cell preadaptation to fucose might allow to overcome lactose acidification. Because fucose is also transported with a proton, it is likely that the acid response system of $E$. coli can be activated during bacteria exposure to fucose ${ }^{33}$.

To the best of our knowledge, this is the first demonstration of a simple and easy quantification method of 2'-FL using a whole-cell biosensor (Table S3). Our biosensor might be applicable for high-throughput screening applications using 96- or 384-well microplates. It has the potential to improve process development, colony selection, quality management, $2^{\prime}$-FL kit development and other steps involved in the production 2'-FL.

\section{Material and methods}

Chemicals and materials. 2'-FL was purchased from AP Technology (Suwon, Korea). L-Fucose was purchased from Carbosynth (Compton, Berkshire, UK). Lactose, trace elements for Riesenberg medium ( $R$ medium) and antibiotics were purchased from Sigma-Aldrich (St. Louis, MO, USA).

Strains and plasmids. The list of strains and plasmids used in this study are listed in Table 1 . The gene encoding $\alpha$-L-fucosidase from Xanthomonas manihotis was synthesized from IDT (Coralville, IA, USA) ${ }^{29,35}$. The synthesized gene was cloned into the pET28b expression vector (Invitrogen, Carlsbad, CA, USA). Next, T7 promoter of pET28b was substituted with the J23100 promoter (BBa_J23100, https://parts.igem.org/Promoters/ Catalog/Anderson) to construct pConFUC. pBbA5aRFP was purchased from Addgene (Addgene_35280) ${ }^{36}$. The lac UV5 promoter of pBbA5aRFP plasmid was also substituted with the J23100 promoter to construct pConRFP that constitutively expressed RFP. To construct pET-ConFUC, the kanamycin resistance gene of pConFUC was substituted for the ampicillin resistance gene amplified from pETduet (Novagen). pColA-ConRFP was constructed by inserting expression regions from pConRFP (Promoter-RBS-CDS) into pColAduet (Novagen).

Determination of 2'-FL cleavage by $\alpha$-L-fucosidase. For the 2'-FL cleavage assay, cells harbouring the pConFUC plasmid were cultured in a $1 \mathrm{~L}$ baffled flask containing $200 \mathrm{ml}$ of Luria-Bertani (LB) medium (1\% 
tryptone, $0.5 \%$ yeast extract, and $1 \%$ sodium chloride). When the optical density (OD) at $600 \mathrm{~nm}$ reached 0.5 , culture was proceeded at $16^{\circ} \mathrm{C}$ and $120 \mathrm{rpm}$ for $16 \mathrm{~h}$. Cells were harvested and the pellet was disrupted using an ultrasonic processor (20\% amplitude, cycles of $1 \mathrm{~s} \mathrm{ON}$ and $1 \mathrm{~s} \mathrm{OFF}$ for $1 \mathrm{~m} 30 \mathrm{~s}$, on ice). Cell debris and insoluble proteins were removed by centrifugation at $15,000 \times g$ for $1 \mathrm{~h}$. The supernatant was collected and concentrated using Centricon 10 (Amicon Co., Beverly, MA, USA). A $5 \mathrm{~g} / \mathrm{L}$ of concentrated supernatant was mixed with $2^{\prime}$-FL in $\mathrm{R}$ medium. The reaction was performed at $37^{\circ} \mathrm{C}$ in the shaking incubator. The reaction mixture was centrifuged at $15,000 \times g$ for $10 \mathrm{~min}$, and the supernatant was filtered using $0.45 \mu \mathrm{m}$ syringe filter.

Detection of $2^{\prime}$-FL from other carbon sources. E. coli cells were pre-cultured in LB medium at $37^{\circ} \mathrm{C}$ by shaking at $250 \mathrm{rpm}$ for $16 \mathrm{~h}$. Pre-cultured cells were harvested and washed three times with sterilized phosphate-buffered saline (PBS, $137 \mathrm{mM} \mathrm{NaCl}, 2.7 \mathrm{mM} \mathrm{KCl}, 10 \mathrm{mM} \mathrm{Na}_{2} \mathrm{SO}_{4}, 1.8 \mathrm{mM} \mathrm{KH}_{2} \mathrm{PO}_{4}$, pH 7.4). Cells were inoculated into $\mathrm{R}$ medium composed of $4 \mathrm{~g} / \mathrm{L}\left(\mathrm{NH}_{4}\right)_{2} \mathrm{HPO}_{4}, 13.5 \mathrm{~g} / \mathrm{L} \mathrm{KH}_{2} \mathrm{PO}_{4}, 1.7 \mathrm{~g} / \mathrm{L}$ citric acid, $1.4 \mathrm{~g} / \mathrm{L} \mathrm{MgSO}$, and $10 \mathrm{~mL} / \mathrm{L}$ trace metal solution, i.e., $10 \mathrm{~g} / \mathrm{L} \mathrm{FeSO}_{4}, 2.25 \mathrm{~g} / \mathrm{L} \mathrm{ZnSO}_{4}, 1.0 \mathrm{~g} / \mathrm{L} \mathrm{CuSO}_{4}, 0.5 \mathrm{~g} / \mathrm{L} \mathrm{MnSO}_{4}, 0.23 \mathrm{~g} / \mathrm{L}$ $\mathrm{Na}_{2} \mathrm{~B}_{4} \mathrm{O}_{7}, 2.0 \mathrm{~g} / \mathrm{L} \mathrm{CaCl}_{2}$, and $0.1 \mathrm{~g} / \mathrm{L}\left(\mathrm{NH}_{4}\right)_{6} \mathrm{Mo}_{7} \mathrm{O}_{24}$, containing various carbon sources ${ }^{37}$. OD and fluorescence intensity were measured during time course (Molecular Devices, Sunnyvale, CA, USA).

Analytical method. Cell concentration was measured by observing the OD at $600 \mathrm{~nm}$. The fluorescence intensity of RFP was measured using the excitation at $584 \mathrm{~nm}$ and emission at $615 \mathrm{~nm}$. The OD and fluorescence intensity were measured using the spectrophotometer (Spectramax M2, Union City, CA, USA). Obtained values in the presence of $2^{\prime}$-FL were divided by the values measured in the absence of $2^{\prime}$-FL to calculate relative optical density (ROD) and relative fluorescence unit (RFU). Concentrations of lactose, L-fucose, and 2'-FL after 2'-FL cleavage were measured by using HPLC (Waters Corporation, Milford, MA, USA) equipped with the Rezex ROA-Organic Acid $\mathrm{H}^{+}$column (Phenomenex, Torrance, CA, USA) and a refractive index (RI) detector. A quantity of $0.01 \mathrm{~N} \mathrm{H}_{2} \mathrm{SO}_{4}$ was used as the mobile phase at a flow rate of $0.6 \mathrm{ml} / \mathrm{min}$ and $50{ }^{\circ} \mathrm{C}^{16}$. All experiments were performed in triplicate.

Statistics. No statistical method was used to determine the sample size in advance. No random method or blind test was used in the experiment and interpretation of results. Numerical analyses were performed using Graph Pad Prism (Graph Pad Software, San Diego, CA, USA).

\section{Data availability}

The datasets generated and/or analyzed during the current study are available from the corresponding author on reasonable request.

Received: 18 March 2020; Accepted: 5 June 2020

Published online: 29 June 2020

\section{References}

1. Bode, L. The functional biology of human milk oligosaccharides. Early Hum. Dev. 91, 619-622 (2015).

2. Bode, L. Human milk oligosaccharides: Every baby needs a sugar mama. Glycobiology 22, 1147-1162 (2012).

3. Walker, W. A. \& Iyengar, R. S. Breast milk, microbiota, and intestinal immune homeostasis. Pediatr. Res. 77, 220-228 (2015).

4. Yu, Z. T. et al. The principal fucosylated oligosaccharides of human milk exhibit prebiotic properties on cultured infant microbiota. Glycobiology 23, 169-177 (2013).

5. Coppa, G. V. et al. Human milk oligosaccharides inhibit the adhesion to Caco-2 cells of diarrheal pathogens: Escherichia coli, Vibrio cholerae, and Salmonella fyris. Pediatr. Res. 59, 377-382 (2006).

6. Thurl, S. et al. Variation of human milk oligosaccharides in relation to milk groups and lactational periods. Br. J. Nutr. 104, 1261-1271 (2010).

7. Goehring, K. C., Kennedy, A. D., Prieto, P. A. \& Buck, R. H. Direct evidence for the presence of human milk oligosaccharides in the circulation of breastfed infants. PLOS ONE 9, e101692 (2014).

8. Gonia, S. et al. Human milk oligosaccharides inhibit Candida albicans invasion of human premature intestinal epithelial cells. J. Nutr. 145, 1992-1998 (2015).

9. Morrow, A. L. et al. Human milk oligosaccharide blood group epitopes and innate immune protection against campylobacter and calicivirus diarrhea in breastfed infants. Adv. Exp. Med. Biol. 554, 443-446 (2004).

10. Vandenplas, Y. et al. Human milk oligosaccharides: 2 '-fucosyllactose (2'-FL) and lacto- $N$-neotetraose (LNnT) in infant formula. Nutrients 10, 1161 (2018).

11. Kunz, C., Rudloff, S., Baier, W., Klein, N. \& Strobel, S. Oligosaccharides in human milk: Structural, functional, and metabolic aspects. Annu. Rev. Nutr. 20, 699-722 (2000).

12. Huang, D. et al. Metabolic engineering of Escherichia coli for the production of 2'-fucosyllactose and 3-fucosyllactose through modular pathway enhancement. Metab. Eng. 41, 23-38 (2017).

13. Chin, Y. W., Seo, N., Kim, J. H. \& Seo, J. H. Metabolic engineering of Escherichia coli to produce 2'-fucosyllactose via salvage pathway of guanosine 5'-diphosphate (GDP)-l-fucose. Biotechnol. Bioeng. 113, 2443-2452 (2016).

14. Chin, Y.-W., Kim, J.-Y., Kim, J.-H., Jung, S.-M. \& Seo, J.-H. Improved production of 2 '-fucosyllactose in engineered Escherichia coli by expressing putative $\alpha-1,2$-fucosyltransferase WcfB from Bacteroides fragilis. J. Biotechnol. 257, 192-198 (2017).

15. Lee, W. H. et al. Whole cell biosynthesis of a functional oligosaccharide, 2'-fucosyllactose, using engineered Escherichia coli. Microb. Cell Fact. 11, 48-56 (2012).

16. Chin, Y. W., Kim, J. Y., Lee, W. H. \& Seo, J. H. Enhanced production of 2'-fucosyllactose in engineered Escherichia coli BL21star (DE3) by modulation of lactose metabolism and fucosyltransferase. J. Biotechnol. 210, 107-115 (2015).

17. Yu, J. et al. Engineering of alpha-1,3-fucosyltransferases for production of 3-fucosyllactose in Escherichia coli. Metab. Eng. 48, 269-278 (2018).

18. Park, Y. et al. Plasmid display for stabilization of enzymes inside the cell to improve whole-cell biotransformation efficiency. Front. Bioeng. Biotech. 7, 444 (2020).

19. Seydametova, E., Shin, J., Yu, J. \& Kweon, D. H. A simple enzymatic method for quantitation of 2'-fucosyllactose. J. Microbiol. Biotechn. 28, 1141-1146 (2018). 
20. Seydametova, E. et al. Development of a quantitative assay for 2'-fucosyllactose via one-pot reaction with alpha-1,2-fucosidase and l-fucose dehydrogenase. Anal. Biochem. 582, 113358 (2019).

21. Petschacher, B. \& Nidetzky, B. Biotechnological production of fucosylated human milk oligosaccharides: Prokaryotic fucosyltransferases and their use in biocatalytic cascades or whole cell conversion systems. J. Biotechnol. 235, 61-83 (2016).

22. Albermann, C., Piepersberg, W. \& Wehmeier, U. F. Synthesis of the milk oligosaccharide 2 '-fucosyllactose using recombinant bacterial enzymes. Carbohydr. Res. 334, 97-103 (2001).

23. Gokhale, U. B., Hindsgaul, O. \& Palcic, M. M. Chemical synthesis of GDP-fucose analogs and their utilization by the Lewis alpha (1-4) fucosyl-transferase. Can. J. Chem. 68, 1063-1071 (1990).

24. Nunez, H. A., Oconnor, J. V., Rosevear, P. R. \& Barker, R. The synthesis and characterization of alpha-L-fucopyranosyl and betafucopyranosyl phosphates and GDP fucose. Can. J. Chem. 59, 2086-2095 (1981).

25. Chaturvedi, P. et al. Fucosylated human milk oligosaccharides vary between individuals and over the course of lactation. Glycobiology 11,365-372 (2001).

26. Chaturvedi, P., Warren, C. D., RuizPalacios, G. M., Pickering, L. K. \& Newburg, D. S. Milk oligosaccharide profiles by reversedphase HPLC of their perbenzoylated derivatives. Anal. Biochem. 251, 89-97 (1997).

27. Bao, Y. W., Chen, C. \& Newburg, D. S. Quantification of neutral human milk oligosaccharides by graphitic carbon high-performance liquid chromatography with tandem mass spectrometry. Anal. Biochem. 433, 28-35 (2013).

28. Thurl, S., MullerWerner, B. \& Sawatzki, G. Quantification of individual oligosaccharide compounds from human milk using highpH anion-exchange chromatography. Anal. Biochem. 235, 202-206 (1996).

29. Wongmadden, S. T. \& Landry, D. Purification and characterization of novel glycosidases from the bacterial genus Xanthomonas. Glycobiology 5, 19-28 (1995).

30. Boronat, A. \& Aguilar, J. Metabolism of l-fucose and l-rhamnose in Escherichia coli: Differences in induction of propanediol oxidoreductase. J. Bacteriol. 147, 181-185 (1981).

31. Tolia, N. H. \& Joshua-Tor, L. Strategies for protein coexpression in Escherichia coli. Nat Methods 3, 55-64 (2006).

32. Patel, L., Garcia, M. L. \& Kaback, H. R. Direct measurement of lactose/proton symport in Escherichia coli membrane vesicles: further evidence for the involvement of histidine residue(s). Biochem.-US 21, 5805-5810 (1982).

33. Bradley, S. A., Tinsley, C. R., Muiry, J. A. \& Henderson, P. J. Proton-linked l-fucose transport in Escherichia coli. Biochem. J. 248, 495-500 (1987).

34. Dykhuizen, D. \& Hartl, D. Transport by the lactose permease of Escherichia coli as the basis of lactose killing. J. Bacteriol. 135, 876-882 (1978).

35. Liu, J. J. et al. Biosynthesis of a functional human milk oligosaccharide, 2'-fucosyllactose, and 1-fucose using engineered Saccharomyces cerevisiae. ACS Synth. Biol. 7, 2529-2536 (2018).

36. Lee, T. S. et al. BglBrick vectors and datasheets: A synthetic biology platform for gene expression. J. Biol. Eng 5, 12 (2011).

37. Riesenberg, D. High-cell-density cultivation of Escherichia coli. Curr. Opin. Biotech. 2, 380-384 (1991).

\section{Acknowledgements}

This research was supported by the Basic Science Research Program through the National Research Foundation of Korea (NRF), funded by the Ministry of Education (2017R1A6A1A03015642 and NRF-2017R1A2B2008211).

\section{Author contributions}

J.S., J.W.L., Y.-S.J., W.-K.M., and D.-H.K. designed research. J.S. performed the experiments. J.S., M.P., C.K., H.K., Y.P., C.B., J.-W.Y., C.-S.S., J.W.L., Y.-S.J., Y.-C.P., W.-K.M. and D.-H.K. analyzed the data. J.S., W.-K.M., and D.-H.K. wrote the manuscript.

\section{Competing interests}

The authors declare no competing financial interest.

\section{Additional information}

Supplementary information is available for this paper at https://doi.org/10.1038/s41598-020-67359-x.

Correspondence and requests for materials should be addressed to W.-K.M. or D.-H.K.

Reprints and permissions information is available at www.nature.com/reprints.

Publisher's note Springer Nature remains neutral with regard to jurisdictional claims in published maps and institutional affiliations.

Open Access This article is licensed under a Creative Commons Attribution 4.0 International

License, which permits use, sharing, adaptation, distribution and reproduction in any medium or format, as long as you give appropriate credit to the original author(s) and the source, provide a link to the Creative Commons license, and indicate if changes were made. The images or other third party material in this article are included in the article's Creative Commons license, unless indicated otherwise in a credit line to the material. If material is not included in the article's Creative Commons license and your intended use is not permitted by statutory regulation or exceeds the permitted use, you will need to obtain permission directly from the copyright holder. To view a copy of this license, visit http://creativecommons.org/licenses/by/4.0/.

(c) The Author(s) 2020 